\title{
An Algorithm for Generating the Sphere Coordinates in a Three-Dimensional Osculatory Packing
}

\author{
By David W. Boyd*
}

\begin{abstract}
This paper develops an efficient algorithm which generates the pentaspherical coordinates of the spheres in an osculatory packing of the three-dimensional unit sphere. The algorithm has a tree-like structure and is easily modified so that, given a prescribed bound, it counts the number of spheres in the packing whose curvatures are less than this bound. The algorithm has been used to produce heuristic estimates of the exponent $M$ of the packing, and these indicate that $M$ is approximately 2.42 .
\end{abstract}

1. Introduction. In a recent paper [3], we described a process for generating the pentaspherical coordinates of all the spheres in an osculatory packing of a threedimensional unit sphere. The procedure given there is not practical because each sphere in the packing is generated infinitely often, and hence this would be a very inefficient way of actually generating the coordinates, requiring a storage of all the coordinates and a comparison of each newly generated coordinate vector with those already stored. In this paper, we present an algorithm which produces the coordinates of each sphere once and only once. Moreover, the structure of the algorithm is tree-like and hence presents no storage problems. We describe how the (theoretically infinite) algorithm can be modified to count the number of spheres in the packing of a given curvature, whose curvatures are less than a prescribed bound. The results of a computer implementation of this algorithm are given, which give heuristic estimates for the exponent $M$ of the packing, using the technique which Melzak [5] used to estimate the two-dimensional constant $S$. These results indicate that $M \approx 2.42$.

2. Preliminary Definitions and Results. We refer the reader to [3] for a more complete explanation of the results mentioned in this section. We begin with a unit sphere (ball) $U$, and four open spheres $S_{1}, \cdots, S_{4}$ which are externally tangent to one another and internally tangent to $U$. An osculatory packing of $U$ beginning with $S_{1}, \cdots, S_{4}$ is a sequence $C=\left\{S_{n}\right\}$ of disjoint open spheres chosen so that $S_{n}$ has the largest radius of spheres contained in $U \backslash\left(S_{1} \cup \cdots \cup S_{n-1}\right)$, for $n=5,6, \cdots$. The exponent of the packing $C$ is the number

$$
M=\sup \left\{t: \sum r_{n}^{t}=\infty\right\}=\inf \left\{t: \sum r_{n}^{t}<\infty\right\},
$$

where $r_{n}$ is the radius of $S_{n}$. In some cases, there is a unique such packing (up to the

Received December 27, 1971.

AMS (MOS) subject classifications (1970). Primary 52A45, 52-04; Secondary 50B99, $50 \mathrm{D} 45$.

Key words and phrases. Sphere packing, osculatory packing, exponent of packing, algorithm, computer study.

* Supported in part by NRC grant A8128. 
order in which spheres of equal radii are listed), and in other cases there are many such packings. Our algorithm produces a specific packing of this type, and it is known (see [3]) that $M$ does not depend on the radii of the spheres $S_{1}, \cdots, S_{4}$. The best known estimates for $M$ are

$$
2.03<M<2.8228 \cdots=(3+\sqrt{ } 7) / 2 .
$$

See Larman [4] for the lower bound and Boyd [1] for the upper bound.

The curvature of a sphere is the reciprocal of its radius. We shall consider the exterior of $U$ as a sphere $X_{1}$ with negative radius and curvature -1 . We shall denote the spheres $S_{1}, \cdots, S_{4}$ alternatively by $X_{2}, \cdots, X_{5}$. Given any two spheres $X$ and $Y$ with radii $r, s$ and whose centres are at distance $d$ apart, we define the separation of $X$ and $Y$ by

$$
\Delta(X, Y)=\left(d^{2}-r^{2}-s^{2}\right) / 2 r s .
$$

Then $\Delta(X, X)=-1$ and $\Delta(X, Y)=1$, if $X$ and $Y$ are externally tangent. One can coordinatize any sphere $Y$ in terms of $X_{1}, \cdots, X_{5}$ by the separation vector

$$
c(Y)=\left(\Delta\left(Y, X_{1}\right), \cdots, \Delta\left(Y, X_{5}\right)\right)^{T},
$$

where the superscript $T$ denotes transpose.

We let $\Delta$ denote the $5 \times 5$ matrix $\left(\Delta\left(X_{i}, X_{j}\right)\right)$ so that $\Delta=J-2 I$, where $J$ is the matrix all of whose entries are 1 , and $I$ is the identity matrix. We have also that

$$
\Delta^{-1}=6^{-1}(J-3 I) \text {. }
$$

The pentaspherical coordinate vector of $Y$ in terms of $X_{1}, \cdots, X_{5}$ is the vector

$$
a(Y)=\Delta^{-1} c(Y) \text {. }
$$

From $a(Y)$, one can obtain the cartesian equations of $Y$. Suppose $X_{i}$ has centre $\gamma_{i}$ and radius $r_{i}$, and $\xi=\left(\xi_{1}, \xi_{2}, \xi_{3}\right)$, and $X_{i}$ has the equation $x_{i}(\xi)=0$, where

$$
x_{i}(\xi)=\left(2 r_{i}\right)^{-1}\left(\left|\xi-\gamma_{i}\right|^{2}-r_{i}^{2}\right)
$$

Then $Y$ has the equation $y(\xi)=0$, where

$$
y(\xi)=\sum\left\{a_{i}(Y) x_{i}(\xi): i=1, \cdots, 5\right\},
$$

and $y(\xi)$ has a form analogous to (7).

The curvature $\eta$ of $Y$ is determined from the curvatures $\epsilon_{1}, \cdots, \epsilon_{5}$ of $X_{1}, \cdots, X_{5}$ by

$$
\eta=a(Y)^{T} \epsilon,
$$

where $\epsilon$ is the column vector $\left(\epsilon_{1}, \cdots, \epsilon_{5}\right)^{T}$.

The vectors $c(Y), a(Y)$ and $\epsilon$ satisfy the equations

$$
\begin{aligned}
c(Y)^{T} \Delta^{-1} c(Y) & =-1, \\
a(Y)^{T} \Delta a(Y) & =-1, \\
\epsilon^{T} \Delta^{-1} \epsilon & =0 .
\end{aligned}
$$

Equation (12) can obviously be written as

$$
\left(\epsilon_{1}+\cdots+\epsilon_{5}\right)^{2}=3\left(\epsilon_{1}^{2}+\cdots+\epsilon_{5}^{2}\right),
$$


in which form it is often known as "Soddy's formula," being named after a poem by Soddy which appeared in 1936. However, the result appears in a much earlier paper (1886) by Lachlan (see [3] for precise references).

The sphere generating process described in [3] generates 5-tuples of spheres $X_{1}(\alpha), \cdots, X_{5}(\alpha)$, where $\alpha$ is any vector with a finite number of components, all in the set $\{1,2,3,4,5\}$. We call the set of such vectors $G$. By convention, $G$ includes a single vector with no components. We let $\mathcal{G}=\left\{X_{i}(\alpha): i=1, \cdots, 5, \alpha \in G\right\}$, and $\mathcal{G}^{\prime}=\mathcal{G} \backslash\left\{X_{1}\right\}$. Theorem 10 of [3] shows that the collection $\mathcal{G}^{\prime}$ is an osculatory packing of $U$. For our purposes, we need only know that the pentaspherical coordinates of the spheres $X_{1}(\alpha), \cdots, X_{5}(\alpha)$ are given by the five columns of a matrix $A(\alpha)$ whose calculation we will now describe. Let $e_{i}(i=1, \cdots, 5)$ be the column vector with all components zero except for a 1 in the $i$ th position, and let $e$ be a vector with all components 1 . Let $A_{i}, i=1, \cdots, 5$, be the following matrices:

$$
\begin{aligned}
& A_{1}=\left(e_{2}, e_{3}, e_{4}, e_{5}, e-2 e_{1}\right), \\
& A_{2}=\left(e_{1}, e_{3}, e_{4}, e_{5}, e-2 e_{2}\right), \\
& A_{3}=\left(e_{1}, e_{2}, e_{4}, e_{5}, e-2 e_{3}\right), \\
& A_{4}=\left(e_{1}, e_{2}, e_{3}, e_{5}, e-2 e_{4}\right), \\
& A_{5}=\left(e_{1}, e_{2}, e_{3}, e_{4}, e-2 e_{5}\right) .
\end{aligned}
$$

If $\alpha=\left(i_{1}, \cdots, i_{m}\right) \in G$, then

$$
A(\alpha)=A_{i_{1}} \cdots A_{i_{m}} .
$$

Note that the entries of the $A_{i}$ and hence $A(\alpha)$ are integers. The columns of $A(\alpha)$, by Lemma 2 of [3], are the pentaspherical coordinates of $X_{1}(\alpha), \cdots, X_{5}(\alpha)$.

In order to generate $G$ as efficiently as possible, we wish to produce an algorithm which will generate each $X \in \mathcal{G}$ once and only once. Observing the formation of the matrices $A_{i}$, we see that if $(\alpha, i)=\left(i_{1}, \cdots, i_{m}, i\right)$, then the sets $\left(X_{1}(\alpha), \cdots, X_{5}(\alpha)\right)$ and $\left(X_{1}(\alpha, i), \cdots, X_{5}(\alpha, i)\right)$ have four spheres in common, and $X_{5}(\alpha, i)$ is a "new" sphere. Thus, apart from the initial spheres $X_{2}, \cdots, X_{5}$, we need only generate the spheres $X_{5}(\alpha)$ for each $\alpha \in G$. It can be shown, with some effort, that the spheres $X_{5}(\alpha)$, with $\alpha$ having components only in the set $\{1,2,3\}$, are all distinct. However, $A_{4}^{6}=I$ and $A_{5}^{2}=I$; so there are many relations, in general, of the form $X_{5}(\alpha)=X_{5}(\beta)$, with $\alpha \neq \beta, \alpha, \beta \in G$. Hence, the "natural" algorithm which would produce all $X_{5}(\alpha)$ with $\alpha \in G_{m}$, successively, for $m=1,2, \cdots$, would be extremely inefficient. Furthermore, in practice, one wants an algorithm which produces all $X_{5}(\alpha)$ whose curvatures lie below some prescribed bound, and the wide variation in curvatures among the $X_{5}(\alpha)$ with $\alpha \in G_{m}$, for some particular $m$, would make this naive method even more unsuitable.

3. An Efficient Algorithm. In this section, we describe an algorithm which produces all the desired coordinates once and only once. We begin with a preliminary version (Lemma 1) which is not quite as efficient as the final version described in Theorem 2, since it produces some coordinates twice. However, with the preliminary algorithm, it is quite easy to see that all coordinates are produced, while this would not be as obvious for the improved algorithm. 
Let $\mathscr{K}$ denote the set of coordinate vectors $a(X)$ for $X \in \mathcal{G}=\left\{X_{i}(\alpha): i=1, \cdots, 5\right.$, $\alpha \in G\}$. It is easily seen that if $a$ is in $\mathcal{K}$, then any vector $a^{\prime}$, whose components are permutations of the components of $a$, is also in $\mathcal{K}$. In particular, if $a^{*}$ is the rearrangement of $a$ into decreasing order, then $a^{*}$ is in $\mathscr{K}$. Hence, it suffices to generate only those vectors whose components are in decreasing order, and we denote this set of vectors by $\mathfrak{K}^{*}$. If $w \in \mathfrak{K}^{*}$, we shall write

$$
U_{i} w=\left(A_{i} w\right)^{*},
$$

so that $U_{i} w$ has components $w_{j}+w_{i}(j \neq i)$ and $-w_{i}$, arranged in decreasing order.

It is clear that each $w \in \mathfrak{K}^{*}$ is of the form $w=U_{i_{1}} \cdots U_{i_{m}} e_{1}$, where $i_{1}, \cdots, i_{m}$ are suitable integers in $\{1, \cdots, 5\}$. We may now think of generating $\mathcal{K}^{*}$ as a list, in the following way: We begin with $e_{1}$, then add to our list $U_{5} e_{1}, \cdots, U_{1} e_{1}$, then repeat with each of the new vectors in our list, and so on. However, if $w$ is in the list and $w_{i}=0$, it is clear that $U_{i} w=w$, so we need not apply $U_{i}$. Also if $w_{i}=w_{i+1}$, we need not apply $U_{i}$. Finally, if $x=U_{i} w$ has just been obtained, and $x_{i}=-w_{i}$, then $U_{i} x=w$, so we need not apply $U_{i}$ to $x$. We shall see in Lemma 1 that we need never apply $U_{i}$ to $w$ if $w_{i}<0$. This will mean $U_{5}$ is never used.

The reader may verify that the following is the beginning of the list which we obtain (writing the coordinate vectors now as row vectors):

$$
\begin{array}{rrrrr}
1 & 0 & 0 & 0 & 0 \\
1 & 1 & 1 & 1 & -1 \\
2 & 2 & 2 & 0 & -1 \\
4 & 4 & 2 & 1 & -2 \\
5 & 5 & 3 & -1 & -1 \\
6 & 6 & 3 & 0 & -2 \\
8 & 6 & 5 & 2 & -4
\end{array}
$$

The number in parenthesis indicates the number of operations $U_{i}$ required to obtain the given vector.

LEMMA 1. Let $U_{1}, \cdots, U_{5}$ be the operations described in the above paragraphs. Given any vector $w=\left(w_{1}, \cdots, w_{5}\right)$, with $w_{1} \geqq \cdots \geqq w_{5}$, we say that $U_{i}$ is admissible for $w$, if $w_{i}>0$ and $w_{i}>w_{i+1}$. Let $U(w)$ be the set of (at most five) vectors $U_{i} w$ such that $U_{i}$ is admissible for $w$. Let $P_{0}=\{(1,0,0,0,0)\}$, and $P_{n}=U\left(P_{n-1}\right)$ for $n=1,2, \cdots$. Then, for $n \geqq 1$,

(a) if $w \in P_{n}$, then $w_{1} \geqq w_{2} \geqq w_{3}>0>w_{5}$;

(b) for each $w \in P_{n}$, if $w_{4} \geqq 0$ or if $0>w_{4}=w_{5}$, then there is a unique $u \in P_{n-1}$ and $a$ unique $i$ so that $U_{i}$ is admissible for $u$ and $U_{i} u=w$;

(c) if $w \in P_{n}$ and $0>w_{4}>w_{5}$, then there are exactly two vectors $u, v \in P_{n-1}$, and corresponding $i$, $j$ so that $w=U_{i} u$ and $w=U_{i} v$;

(d) if $m, n \geqq 0$, and $m \neq n$, then $P_{m}$ and $P_{n}$ are disjoint.

Proof. We shall give the proofs by induction, proving (d) in the form that $P_{m} \cap P_{n}=\varnothing$ if $m<n$. The results are true, by inspection, if $n=1$. To prove (a) for $P_{n}$, let $w \in P_{n}$, and $w=U_{i} u$ with $u \in P_{n-1}$ and $U_{i}$ admissible for $u$. Then $u_{i}>0$, and the components of $w$ are $u_{i}+u_{i}(j \neq i)$ and $-u_{i}$ in decreasing order. Since 
(a) holds for $u$ (by induction), we at least have $w_{1} \geqq w_{2}>0$, so $-u_{i}$ is one of $w_{3}, w_{4}$ or $w_{5}$. Now, by checking all possibilities for $i$, we can see that

$$
w_{3}+w_{4}+w_{5} \geqq u_{3}+u_{4}+u_{5} .
$$

Then it follows by induction that $w_{3}+w_{4}+w_{5} \geqq 1$, and hence certainly $w_{3}>0$. Since at least one component of $w$ (namely $-u_{i}$ ) is negative, certainly $w_{5}<0$.

To prove (b), let $w, u, i$ be as above. By assumption, $w$ has but one distinct negative component, so it must be $-u_{i}$. That is, $w_{5}=-u_{i}$ and the other components of $u$ must be $w_{1}+w_{5}, \cdots, w_{4}+w_{5}$. Since $u$ is in decreasing order, $u$ is determined uniquely. The number $i$ is also unique since $U_{j} u=U_{i} u$ for $j<i$ would contradict the admissibility of $U_{i}$ for $u$.

To prove (c), suppose $w=(a, b, c,-d,-e)$, where $0<d<e$. We find, by extending the list preceding this lemma that $n$ must be $\geqq 6$. There are two possibilities (and we show both hold).

(i) $d=u_{i}$ for some $u \in P_{n-1}$,

(ii) $e=v_{i}$ for some $v \in P_{n-1}$.

If (i) holds, then

$$
u=(a-d, b-d, c-d, d,-d-e)^{*} \in P_{n-1} .
$$

By (b) or (c) for $u, u$ has a predecessor in $P_{n-2}$ which we may take to be

$$
u^{\prime}=(a-2 d-e, b-2 d-e, c-2 d-e, d+e,-e)^{*} \in P_{n-2},
$$

and, by the same argument, $u^{\prime} \in U\left(u^{\prime \prime}\right)$, where

$$
u^{\prime \prime}=(a-2 d-2 e, b-2 d-2 e, c-2 d-2 e, d, e)^{*} \in P_{n-3} .
$$

The vector $u^{\prime \prime}$ is symmetric in $d$ and $e$; however, we can obtain successively

$$
v^{\prime}=(a-d-2 e, b-d-2 e, c-d-2 e, d+e,-d)^{*} \in U\left(u^{\prime \prime}\right) \subset P_{n-2}
$$

and

$$
v=(a-e, b-e, c-e, e,-e-d)^{*} \in U\left(v^{\prime}\right) \subset P_{n-1} .
$$

Finally, we see that $w \in U(v)$. Note that $u \neq v$, for if $u=v$, then since $d \neq e$, we must have $d$ equal to one of $a-e, b-e$ or $c-e$. If, say, $d=a-e$, then $b-e=$ $c-d$ and $c-e=b-d$ are the only possibilities and together these imply $d=e$, a contradiction. Since $w$ has clearly at most two predecessors in $P_{n-1}$, we have proved (c).

Finally, to prove (d), we observe that if $w \in P_{n}, n \geqq 1$, then $w_{5}<0$, so $P_{n} \cap P_{0}=$ $\varnothing$. Now, we use induction on $m$ to show $P_{m} \cap P_{n}=\varnothing$ if $m<n$. For, if $w \in P_{n} \cap$ $P_{m}$, then, by (b) or (c), $w \in U(u)$, where $u \in P_{n-1}$ and also $u \in P_{m-1}$. Thus $P_{n-1} \cap$ $P_{m-1} \neq \varnothing$, a contradiction of the induction assumption.

THEOREM 2. For any vector $w=\left(w_{1}, \cdots, w_{5}\right)$, let $T_{i}(i=1, \cdots, 4)$ be the (linear) mapping which maps $w$ into the vector $w^{\prime}$, where

$$
\begin{aligned}
w_{i}^{\prime} & =w_{i}+w_{i} & & \text { if } j<i, \\
& =w_{j+1}+w_{i} & & \text { if } i \leqq j<5, \\
& =-w_{i} & & \text { if } j=5 .
\end{aligned}
$$


We say that $T_{i}$ is admissible for $w$ under the following circumstances:

(i) if $i=1,2,3$, we require $w_{i}>w_{i+1}$,

(ii) if $i=4$, we require $2 w_{4}+w_{5} \geqq 0$ and $w_{4}>0$.

Let $T(w)$ be the set of (at most four) vectors $T_{i} w$ such that $T_{i}$ is admissible for $w$. Let $Q_{0}=\{(1,0,0,0,0)\}$ and $Q_{n}=T\left(Q_{n-1}\right)$ for $n=1,2, \cdots$. Then $Q_{n}=P_{n}(n=0,1, \cdots)$, where $P_{n}$ is as in Lemma 1. Also, for each $w \in Q_{n}$, there is a unique $u \in Q_{n-1}$ and $a$ unique $i$ for which $T_{i}$ is admissible for $u$ and $w=T_{i} u$.

Suppose that $X \in \mathcal{G}$ and has the coordinate vector $a=a(X)$. Then, there is a unique $n$ and $w \in Q_{n}$ such that $a^{*}=w$. Furthermore, for any $n$, if $w \in Q_{n}$, then all distinct permutations of $w$ are coordinate vectors of spheres in $G$.

Proof. We shall show that $Q_{n}=P_{n}$ of Lemma 1. This is clearly true for $n=0$ and we proceed by induction. Suppose $w \in Q_{n}$ and $w=T_{i} u$, with $u \in Q_{n-1}=P_{n-1}$, and $T_{i}$ is admissible. Then we claim $T_{i} u=\left(T_{i} u\right)^{*}=U_{i} u$. We need only show that $w_{5} \leqq w_{4}$. This holds if and only if $2 u_{i}+u_{5} \geqq 0$. For $i=4$, this is part of requirement (ii) and for $i=1,2,3$ it follows from $u_{3}+u_{4}+u_{5} \geqq 1$ proved in Lemma 1, since

$$
2 u_{i}+u_{5} \geqq u_{i}+u_{4}+u_{5} \geqq u_{3}+u_{4}+u_{5}
$$

for $i=1,2$ or 3 .

Hence, we have $Q_{n} \subset P_{n}$. To show equality, suppose $w \in P_{n}$. Then by Lemma 1 (b), (c), $W$ has one or two predecessors in $P_{n-1}=Q_{n-1}$. In either case, one of the predecessors is

$$
u=\left(w_{1}+w_{5}, \cdots, w_{4}+w_{5},-w_{5}\right)^{*},
$$

and, if $u_{i}=-w_{5}$, then $2 u_{i}+w_{5}=w_{4}-w_{5} \geqq 0$, and $u_{i}>0$, so that if $i$ is chosen maximally, $T_{i}$ is admissible for $u$, and so $w=T_{i} u \in Q_{n}$. It is easy to see that (ii) rules out the other possible predecessor for $w$, so $w$ has a unique predecessor in $Q_{n-1}$.

Finally, our remarks at the beginning of this section prove the final statement of the theorem.

Remarks. 1. We shall call the set of spheres whose coordinates are rearrangements of a vector in $P_{n}=Q_{n}$ the $n$th generation of spheres. It is clear that $n$ is the minimal integer for which $X \in \mathcal{G}$ has a representation as $X_{i}(\alpha)$ where $\alpha$ has $n$ components.

2. There are certain aspects of the algorithm described in Theorem 2 which are of importance in practice. We may assume that the curvatures $\epsilon_{1}, \cdots, \epsilon_{5}$ of $X_{1}, \cdots, X_{5}$ satisfy $-1=\epsilon_{1}<\epsilon_{2} \leqq \cdots \leqq \epsilon_{5}$. In addition to the vector $\epsilon$, we use the vector $\kappa=\Delta^{-1} \epsilon$ which has components

$$
\kappa_{j}=\left(\epsilon_{1}+\cdots+\epsilon_{5}-3 \epsilon_{j}\right) / 6 .
$$

According to (9) and (6), the curvature $\epsilon(X)$ of a sphere $X$ is given by

$$
\epsilon(X)=a(X)^{T} \epsilon=c(X)^{T} \kappa .
$$

We shall assume always that $\kappa_{i} \geqq 0$ for all $j$. The geometrical meaning of this is that the centre of $U$ lies in the convex hull of the centres of $X_{2}, \cdots, X_{5}$. We claim that this condition implies that if $u \in P_{n}(n \geqq 1)$ is the coordinate vector of a sphere $X$, and $w=T_{i} u$ (with $T_{i}$ admissible) corresponds to a sphere $Y$, then the curvatures of $Y$ and $X$ satisfy

$$
\epsilon(Y) \geqq \epsilon(X)
$$


To see this, one notes that $c(X)$ and $c(Y)$ are vectors with components which are integers $\geqq 1$ (since $X \in \mathcal{G}$ is not one of $X_{1}, \cdots, X_{5}$ so $\Delta\left(X, X_{i}\right) \geqq 1$ ). By direct computation,

$$
\begin{aligned}
c_{j}(Y) & =c_{j}(X), & & 1 \leqq j<i, \\
& =c_{j+1}(X), & & i \leqq j<5, \\
& =c_{i}(X)+6 u_{i}, & & j=5,
\end{aligned}
$$

so that $c_{j}(Y) \geqq c_{j}(X)$ for all $j$, from which (20) follows.

Also, note that if $u \in P_{n}$, then the set $T(u)$ represents the coordinates of up to $480=4 \times 5$ ! spheres of $\mathcal{G}$, corresponding to the admissible $T_{1}, \cdots, T_{4}$ and the distinct permutations of each $T_{i} w$. We claim that, of these spheres, the one with least curvature is that with components $T_{i} u$, where $i$ is the largest value for which $T_{i}$ is admissible. To see this, note that $w=T_{i} u$ is in decreasing order, so if $\sigma$ is any permutation of $\{1, \cdots, 5\}$, then $\epsilon_{1} \leqq \cdots \leqq \epsilon_{5}$ implies

$$
\epsilon_{1} w_{1}+\cdots+\epsilon_{5} w_{5} \leqq \epsilon_{1} w_{\sigma(1)}+\cdots+\epsilon_{5} w_{\sigma(5)} .
$$

Also, if $j<i$, and $T_{i}$ is admissible, and if $Y$ and $Z$ are the spheres corresponding to $T_{i} u$ and $T_{i} u$, respectively, then (21) shows that

$$
\epsilon(Y) \leqq \epsilon(Z) .
$$

In practice, one does not permute the vector $w$, but rather, permutes the curvatures $\epsilon_{i}$ since this need be done only once. The various vectors $w$ are weighted as appropriate for the number of distinct permutations. For $n \geqq 3$, if $w \in P_{n}$, it can be shown that there are only three possibilities; either $w_{1}, \cdots, w_{5}$ are distinct or else there is one equal pair of $w_{i}$ or else two such pairs.

3. We mentioned earlier that the algorithm has a tree-like structure. In other words, each $w$ in $\bigcup P_{n}$ has a unique representation in the form

$$
w=T_{i(n)} \cdots T_{i(1)} e_{1},
$$

where $T_{i(k)}$ is admissible for $T_{i(k-1)} \cdots T_{i(1)} e_{1}$ for each $k=1, \cdots, n$. Thus, one can imagine an infinite tree: The nodes at height $n$ correspond to the vectors $w$ in $P_{n}$; edges lead from a $w$ in $P_{n}$ to the vectors in $T(w)$ in $P_{n+1}$. Assigned to each node $w$ in the tree is the number $w^{T} \epsilon=\epsilon_{1} w_{1}+\cdots+\epsilon_{5} w_{5}$ corresponding to the least curvature of the spheres corresponding to that node (by (22)). We set an index $f(n)$ for each level initially at 4 for all $n$, and generate successively $w_{0}=e_{1}, T_{i(0)} w_{0}=w_{1}, T_{i(1)} w_{1}=$ $w_{2}, \cdots$ where $i(n)$ is the largest $i \leqq f(n)$ for which $T_{i}$ is admissible for $w_{i}$. Once $T_{i(n)}$ has been used, we set $f(n)=i(n)-1$. To determine the number of spheres $W(m)$ for which $m \leqq w^{T} \epsilon<m+1$ for all integers $m \leqq B$, say, we proceed upward until $w_{n}^{T} \epsilon>B$. With each new node generated, we increase $W(m)$ by 1 for each sphere corresponding to this node which has curvature $\eta$, with $[\eta]=m$. Once $w_{n}^{T} \epsilon>B$, we reduce $n$ and proceed upward along a new branch corresponding to $f(n)$. If $f(n)=0$, we reduce $n$ further. Eventually, this will generate all spheres. Notice that we need provide storage only for $N$ coordinate vectors, where $N$ is such that for $n \geqq N$, $w^{T} \epsilon>B$ for all $w \in P_{n}$. The value of $N$ is quite small, of the order of magnitude of $\sqrt{ } B$. 
4. Results of a Computer Study. Using Theorem 2 and the succeeding remarks, we wrote a computer programme in FORTRAN which counts the number $W(C)$ of spheres in the packing $\mathcal{G}^{\prime}$ which have the integer part of their curvature equal to $C$ for all $C \leqq$ CMAX, where CMAX is prescribed, as are $\epsilon_{1}=-1, \epsilon_{2}, \cdots, \epsilon_{5}$ satisfying (13), and with $\kappa_{j} \geqq 0$, where $\kappa_{j}$ is given by (17). Using the values of $W(C)$ so obtained, a linear law in $\log C$ is fitted to the cumulative sum $\log \left(\sum W(i): i \leqq C\right)$ by using least squares. In this way, one obtains an approximate relation

$$
\left|\left\{X \in \mathcal{G}^{\prime}: \epsilon(X) \leqq C\right\}\right| \approx A C^{-M^{\prime}},
$$

where $M^{\prime}$ should be an approximation to $M$. In [5], Melzak used a similar method to estimate the two-dimensional packing constant $S$ and obtained the heuristic result

$$
S \approx 1.306951
$$

Since this author has recently [2] shown rigorously that

$$
1.300197<S<1.314534,
$$

the method would seem quite reliable.

For the initial choice of curvatures $(-1,2,2,3,3)$, suggested by Soddy's "bowl of integers" [6], the curvatures of all spheres are clearly integers. In this packing, the two spheres of curvature 2 are tangent along a diameter of $U$ and six spheres of curvature 3 touching $U$ and the two spheres of curvature 2 can be arranged in a ring around the central spheres. (This corresponds to the matrix relation $A_{4}^{6}=I$.) Thus, the packing $\mathcal{G}^{\prime}$ is unchanged by a rotation through $\pi / 3$ about the diameter common to $X_{1}, X_{2}$ and $X_{3}$. This means that $W(C)$ is divisible by 6 for all $C>2$, which is a good check on the computations. For CMAX $=300$, the running time on the IBM $360 / 65$ at the University of British Columbia was 135 seconds. The total number of spheres, with curvature $C$ at most 300, is 305,594 and these occupy .94727 of the volume of $U$. The values of $A$ and $M^{\prime}$ of (24) are $A_{1}=.2988455$ and

$$
M_{1}=2.42009 \text {. }
$$

An interesting point is that in this packing the curvature of each sphere satisfies $C \equiv 0,2(\bmod 3)$. This is easily proved once it is noted (by induction) that all coordinate vectors in $\bigcup P_{n}$, modulo 3 , are rearrangements of one of the three types $(1,1,1,1,2),(0,2,2,2,2)$ or $(0,0,0,0,1)$. The number $N$ mentioned in Remark 3 of the previous section (the "height of the tree") was 38. Much information was printed out which is not reproduced here. In particular, the 300 values $W(C)$ were printed, and the numbers of spheres of curvature at most 300 in each generation $P_{n}, n=$ $1, \cdots, 38$, were printed.

In another run with initial curvatures $(-1,2,2,3,3)$ and CMAX $=100$, we produced a list in lexicographic order of the coordinate vectors $w$, and the corresponding separation vectors.

Another interesting set of initial curvatures is $(-1, a, a, a, a)$ where $a=1+\sqrt{ } 6 / 2$. This corresponds to a configuration in which $X_{2}, \cdots, X_{5}$ have their centres at the vertices of a regular tetrahedron. With CMAX $=600$, running time was 94 seconds. There were 1,693,595 spheres counted, distributed into 49 generations. The values of $A$ and $M^{\prime}$ were $A=.3149755$ and 


$$
M_{2}=2.41748 \text {. }
$$

From (27) and (28), it seems reasonable to expect that $M \approx 2.42$. A rigorous justification of this would be of considerable interest. Even more interesting would be a rigorous justification of the method itself, but this would seem to present considerable difficulties.

Department of Mathematics

The University of British Columbia

Vancouver 8, B. C., Canada

1. D. W. BoyD, "On the exponent of an osculatory packing," Canad. J. Math., v. 23, 1971 , pp. 355-363. MR 42 \#6723.

2. D. W. BoyD, "Improved bounds for the disk-packing constant," Aequationes Math. (To appear.)

3. D. W. Boyd, "The osculatory packing of a three dimensional sphere," Canad. J. Math. (To appear.)

4. D. G. LARMAN, "On the exponent of convergence of a packing of spheres," Mathematika, v. 13, 1966, pp. 57-59. MR 34 \#1928.

5. Z. A. MelzaK, "On the solid-packing constant for circles," Math. Comp., v. 23, 1969, pp. 169-172. MR 39 \#6179.

6. F. SoDDY, "The bowl of integers and the hexlet," Nature, v. 139, 1937, pp. 77-79. 